\title{
Analyzing Mobile Services in Alternative Agrifood Networks
}

\author{
Antonio Palmiro Volpentesta and Marco Della Gala \\ Department of Mechanical Energy and Management Engineering, \\ University of Calabria via P. Bucci, 42\C, 87036 Rende (CS), Italy \\ \{antonio.volpentesta, marco.dellagala\} @unical.it
}

\begin{abstract}
Recently, Alternative Agrifood Networks (AAFNs) represent a form of collaborative agrifood networks characterized by a re-connection and close communication among producers and consumers in order to overcome the limits of dominant capital-intensive agribusiness system. In this paper, we propose a framework to analyze and explore the value of the use of mobile information services in an AAFN. The applicability of the framework is shown by presenting some results obtained from an analysis of different types of case studies referring to the use of mobile services in real-world AAFNs.
\end{abstract}

Keywords: agribusiness, Alternative Agrifood Networks, mobile services, mobile applications.

\section{Introduction}

Over recent years, the industrialization of mainstream food systems has resulted into a concentrated control of product markets, with a small number of dominant organizations. Big organizations monitor every transaction among millions of disconnected producers and consumers, leading both to the loss of decisional power for farmers and producers and to the crisis of confidence in mass-produced, 'placeless and faceless' food products, as well as the 'crisis of trust' among consumers [1], [2]. In addition to that, rural SMEs are subjected to a continuous imbalance of their bargaining power; they suffer cost-price squeeze, unfair contractual agreement, rising production costs and declining commodity prices that reduce their profitability [1], [2] [3], [4].

All the above mentioned factors have induced farmers, and other people or organizations dealing with agricultural and rural issues, to organize themselves spontaneously in order to solve their problems and those of rural communities. This has led to new and alternative business models to guarantee competitive advantages, to improve farm revenue streams, to return in taking an active role in the agrifood ${ }^{1}$ system, and to develop new consumer market niches, [3], [4], [5], [6], [7], [8].

\footnotetext{
${ }^{1}$ Agrifood is a generalized term for mostly farm-originated products that are intended for human consumption. Of course, this definition is a fuzzy definition, since it depends on how much a product is farm-originated. A deep discussion on farm-originated food is beyond the scope of this paper. However, it is useful to distinguish:
} 
With this aim, different forms of collaborative networks have been introduced in the agrifood sector as characterized by a re-connection or close communication among producers and consumers, allowing the development of new forms of relationship and governance of the actors' network and also enhancing a redistribution of value for primary producers [1], [2], [9], [10].

According to Goodman [5], alternative agrifood networks (AAFNs) is an umbrella term that is used to indicate all these new forms of collaborative development. AAFNs are alternative to the logic and organizational arrangements of the dominant agrifood system which is based on long and multinational supply chains in the direction of shortening the distance (physical, social, cultural, and economic) between world production and world consumption [11], [2].

AAFNs can be shaped into different organizational forms (Direct on Farm Sales, Farmers Markets, Box Schema, CSA, Collective Kitchens) in relation to the socioeconomic context of the reference territories and to the peculiarities of the many grassroots initiatives promoted both by producers and consumers, see [12] for a descriptions of these forms.

However, no matter of organizational issues, in any AAFN the agrifood is regarded as 'embedded' with value-laden information, concerning the mode of production, provenance and distinctive quality assets of the product, when it reaches the consumer. An AAFN provides a sort of liminal space that subverts the normal experience of food shopping [13] and where a variety of information and knowledge related to agriculture, rural economy, the environment, food production, healthy eating and consumer values, may be exchanged [14].

Nowadays, such information/knowledge exchange may be supported by new types of services based on recent technology developments. In particular, mobile services can provide information offerings regardless of temporal and spatial constraints, in an AAFN, they make easy to share detailed agrifood and agrifood source information, bringing AAFN people closer to each other. On one hand, agrifood producers can give consumers insight into sourcing and production methods, monitor their customer base, and make transparency a competitive advantage; on the other hand, consumers may be empowered with more timely and accurate information to make better buying decisions based on their personal values. In essence, the use of mobile services may contribute to the growth of AAFNs through supporting grass-roots and up approaches aimed to connect local citizens, restaurants, and produce distributors directly to the local farmers in their communities.

In this paper, we propose an AAFN mobile service analysis framework for eliciting key components that concur to generate value for AAFN people. In our opinion, such a framework constitutes a valid basis to develop an evaluation model of AAFN mobile information services under a user-centric perspective. Its applicability is

primary processed products that are consumed in the original state, as they are produced with no value additions being made, or are processed and refined as little as possible, before being consumed;

secondary processed products that require basic level of processing (grading, sorting, cleaning, cutting, etc.) before they are consumed;

tertiary processed products that result in the output being in a different form, shape and with a higher value as compared to the original production. 
showed by presenting some results obtained from an analysis of different types of case studies referring to the use of mobile services in real-world AAFNs.

\section{AAFN Mobile Information Services}

The term mobile service is used to describe all services that can be used independently of temporal and spatial restraints, and that are accessed through a mobile handset (mobile phone, PDA, PC tablets , smartphone, etc.). They differ "from traditional interpersonal services that are delivered face-to-face, or from other types of e-services, such as wireless online services, where the service delivery is linked to a specific fixed local area network or specific location", [15]. In general, benefits of mobile services are mainly due to four factors: ubiquity, convenience, localization and personalization that differentiate mobile services from online services [16].

In an AAFN, mobile services can provide new opportunities for serving awareness and transparency needs of consumers as well as request of information of producers on their business processes. For example, they may provide farm site or agrifood product information (processing methods, provenance of the produce, agrifood physiological and health aspects, etc.) in digital format (labels, pictures, videos, geo position, and graphics) on a user's individual display or through headphones for audio content. Moreover, the integration of new technologies, such as object recognition, feature tracking, RFIDs, Near Field Communication (NFC), geotagging, and web services, in mobile devices has enabled to conceive new mobile services that enrich the relations between AAFN actors and better support their activities.

AAFN mobile services can be grouped in five major classes [17]:

Virtual Visits Services: most of them provide an interactive virtual farm tour. They aim to create an opportunity for consumers to know the countryside and to facilitate a wider understanding of the environmental, economic and social issues linked to an AAFN. Moreover, they improve consumers' confidence in offered products allowing consumers to find out how agrifood is grown and produced and discover what happens on a farm. For instance, in a virtual egg farm tour consumers can get information about where eggs come from and how hens are raised (in cages or in barns with or without access to outdoors run).

Traceability and Product Related Information Services: they deliver tailor made information (including physiological and health aspects, origin, recipes, conserving methods, etc.) according to individually determined criteria selected by a consumer [18]. Traceability data can be provided by a code. Product information can be got through scanning the related code by using a smartphone camera. For instance, these services enable consumers to read product descriptions, view photos and availability, compare prices, read recipes and access to information such as origin of the product, presence or absence of GMO, absence of pesticides, fat content, food miles, etc..

Location-Based Services and Geospatial Services: based on geotagging technology, they make searching for in season agrifood products and places where AAFN activities may occur. For instance, through GPS routing and getting turn-by-turn directions, they may enable a consumer to easily find farmers markets near his/her 
current location or within a state/city, and to get market information (hours/dates of operation, parking places, prices of products for sale, etc.).

Dietary and Health Services: exploiting location-based services or object recognition applications, they essentially provide a consumer with agrifood nutritional information (e.g. what vitamins a fruit or a vegetable have, and what diseases they are good at protecting against) and dietary advices (e.g. daily healthy-eating tips and how many calories, vitamins, and minerals are in a certain meal). Some of them are customized services that provide personal dietary and health advices (e.g. explanations and warnings about agrifood's nutrients and ingredients) on the base of the consumer's profile (body mass index, intolerances, special diets, alimentary restrictions, etc.).

Social Networking Services: they allow interactive learning experiences through uploading of photos, tagging of photos and presenting of feedback or recommendations. They represent a mean for consumers to get information about their past behavior, or actions of other consumers in similar positions [19]. For instance, consumers, farmers, distributors, and food producers can submit pictures of their products and add information about which farmers' market, restaurant, or grocer the agrifood is bound for; this helps people to know where agrifood comes from, who produces it, and who handles it as it travels from its source to plate.

The overall value of these mobile services is influenced by four main factors:

1. Consumption motivation: this factor refers to the relative importance of the learning, social and utilitarian value generated for service users. Learning value refers to intrinsic motivation that is associated with needs of experiential learning about AAFN agrifood products, people, organization, and environment [12] [20]. Social value is also referred to intrinsic motivation, but the associated needs are more communicative and relational (e.g. sharing downloaded digital content and received messages for AAFN social belongingness). The utilitarian value refers to extrinsic motivation that exists in goal directed service use and is associated with effectiveness/efficiency information needs (e.g. saving time and easily finding vendor or producer location, time table of operations, etc.) in performing a task [15].

2. Use context criticality: the time when and place where the service is used affect the value generated for service users. Temporal criticality points out how urgently the user needs the service. Spatial criticality indicates if the service can be used anywhere, i.e. non-location-based service, or if the service should be used at a specific AAFN place (e.g. a farm site), i.e. location based service.

3. User's role: this factor refers to the relative importance of the role played in the AAFN by a service user. The service can be oriented to specific AAFN roles (e.g. consumer, producer, organization operator, etc.) in order to increase the value gained by its users.

4. Information source: any mobile service utilizes a variety of knowledge and information sources that can be valuable in terms of their extension (or coverage), their intension (or density), and their trustworthiness. Such source qualities naturally affect the value generated by the service for its users.

The above-described factors are further expanded in the next section and have been used as the base in developing our framework for analysing mobile information services in an AAFN. 


\section{A Mobile Service Analysis Framework}

In this section, we propose an AAFN service analysis framework for eliciting key components that concur to generate value for AAFN people. The value segmentation, introduced in the previous section and such a framework constitute a useful basis to develop an evaluation model of AAFN mobile information services under a usercentric perspective. In order to analyze the overall value generated by an AAFN mobile service, three main components are considered:

a. the human-service system interaction (HSSI);

b. the knowledge and information source (KIS);

c. the information content domain (ICD).

Such components are interrelated, as depicted in Figure 1, in the following sense:

HSSI-ICD: the information exchanged in the interaction regards some ICD elements. For instance, a mobile service may provide information about agrifood products (e.g. seasonality or shelf life) or AAFN organization culture (e.g. norms or values).

HSSI-KIS: in the interaction, some KISs are made accessible by the service system. For instance, a mobile application can make it possible to access an organizational database containing data about the sale, movement, and distribution of produce along the AAFN.

KIS-ICD: any KIS of the service system is related to a subset of ICD that constitutes its information content domain. For instance, a research center, as an AAFN external source, may be involved in service management by providing scientific knowledge that can be lately exploited by farmers to learn how to grow better quality, higheryielding crops.

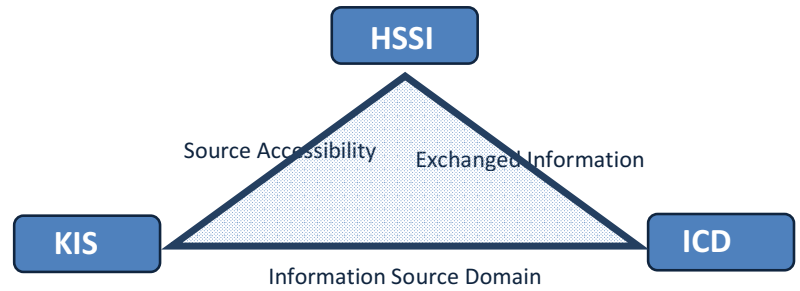

Fig. 1.

Let us discuss now the structure of each single component.

\subsection{Human-Service System Interaction (HSSI)}

By taking into account a user-centric perspective, we group interaction characteristics into dimensions as follows:

a.1 User's role: it specifies the role of the user in the AAFN, when acting in the interaction with the service system. Such role is typed as consumer, producer, or organization operator.

a.2 Scope: it specifies the type of motivation for the participation to AAFN activities. Participation is seen in terms of expressing and discussing ideas, 
developing plans, evaluating actions, and decision-making. Motivation types are social (e.g. tighter relationship with others, social belonging), ecological (e.g. lower environmental impact), economical (e.g. available income/budget impact), and personal wellbeing (e.g. physical and mental health, pleasant time).

a.3 Timing: it specifies the moment in the agrifood chain of the AAFN when the interaction occurs: "production", "distribution", "consumption", and "waste management" turn out to be the values along this dimension. Synchronicity and asynchronicity can be also considered in this group interaction characteristics, especially when the use situation is time critical.

a.4 Place: it specifies the place where the interaction occurs; this place is typed as "farm site", where the agrifood product is coming from, "agrifood terroir", i.e. the land bestowed upon the agrifood, "proximate area", i.e. an area (e.g. urban area) that is close to the agrifood terroir. Such a dimension is particular important in locationbased services in which a mobile application uses the user's location interaction place.

a.5 Flow type: it specifies both the type and the direction of the information flow in the interaction; it is typed as "informational", i.e. information is only pushed from a mobile application to the user, "reporting", i.e. information is only pulled by the mobile application from the user, and "interactional", i.e. a bidirectional flow between the user and the mobile application occurs.

\subsection{Knowledge and Information Source (KIS)}

A service system makes use of many KISs that can be grouped in the following categories:

b.1 Participants: AAFN people (e.g. consumers, producers, FM operators, regarded as individuals) constitute KISs, since they possess interesting information and individual knowledge, independent of an organizational entity's existence, which can be exchanged in a user interaction with the service system. For instance, producers could provide information and knowledge about the farm origin of agrifood, including the environmental and social conditions of its production, as well as the cultural significance behind agrifood tied to a specific method or place of production; consumers could provide information and knowledge about taste, culinary uses and sales responsiveness of agrifood products.

b.2 Organization: any organizational entity (e.g. a consumers group, a group leader, a farmer-driven board of directors, a vendor-consumer advisory committee, a FM operator) in an AAFN is a source of organizational information and knowledge that is embedded within the behaviours that manifests in the overall AAFN organization through its culture (values, principles, norms, traditions, unwritten rules, and informal procedures), its structure (roles, relationships, and regulations that govern their use), and its business function (activities or tasks, such as planning, production, sales, performed together to obtain a defined set of results). For instance, a FM operator could provide information and knowledge about market prices and even latest agricultural practices that are essential in creating opportunities for small producers to know their economic performance.

b.3 Environment: external entities (e.g. input providers, certifying and extension agencies, NGOs, governments, financial service providers, research centers and other 
agrifood organizations) operating in an AAFN surrounding (socio-political, economical, bio-ecological) environments constitute a KIS. For instance, input retailers often serve as a de-facto source of expert information for small farmers who can learn about the application and use of nutrients and pesticides.

\subsection{Information Content Domain (ICD)}

In analyzing the component ICD, we distinguish two main dimensions:

c.1 Content exposure: it refers to what extent the ICD (or a part of it) is accessible to service users. In public exposure the domain is publicly accessible by anyone at any time. In private exposure the domain can only be accessed by a pre-selected (by a third party) group of users.

c.2 Content orientation: it refers to semantic aspects of the ICD. An ICD of an AAFN service may be related with the following categories:

c.2.1 agrifood product, i.e. content items regarding attributes (e.g. price, seasonality, varieties, taste shapes, textures and aromas of agrifood) of products exchanged in an AAFN;

c.2.2 people: content items that are useful to know a person (e.g. trustworthiness, loyalty, integrity, wishes and needs) involved in AAFN activities;

c.2.3 organization functions: content issues about activities (e.g., agricultural practices, processing methods, food preparation) at any stage (production, distribution, consumption, and waste management) of the agrifood chain in an AAFN;

c.2.4 organization structure: content items that are useful to know roles, relationships, and regulations that govern their use in an AAFN;

c.2.5 organization culture: content items that are useful to know norms, values, experiences, and history of an AAFN;

c.2.6 environment: content items regarding social, economic and natural aspects of the environment surrounding an AAFN.

\section{$4 \quad$ Selected Case Studies}

In order to demonstrate the applicability of our service analysis framework, we have selected and analysed 10 real world cases of AAFN services that have been considered relevant in the mobile service classes described in section 2 .

For each of them, the analysis has been carried out by examining relative web sites, combining qualitative information from relative professional literature, and, when possible, downloading and using relative supporting mobile applications.

Analysis results are synthetized in Table 1, where rows correspond to mobile service classes and columns correspond to analysis dimensions (including organizational forms of an AAFN as a further dimension). Mobile service examples mentioned in the table may be placed in several classes depending on the use situation and the individual user's preferences. 


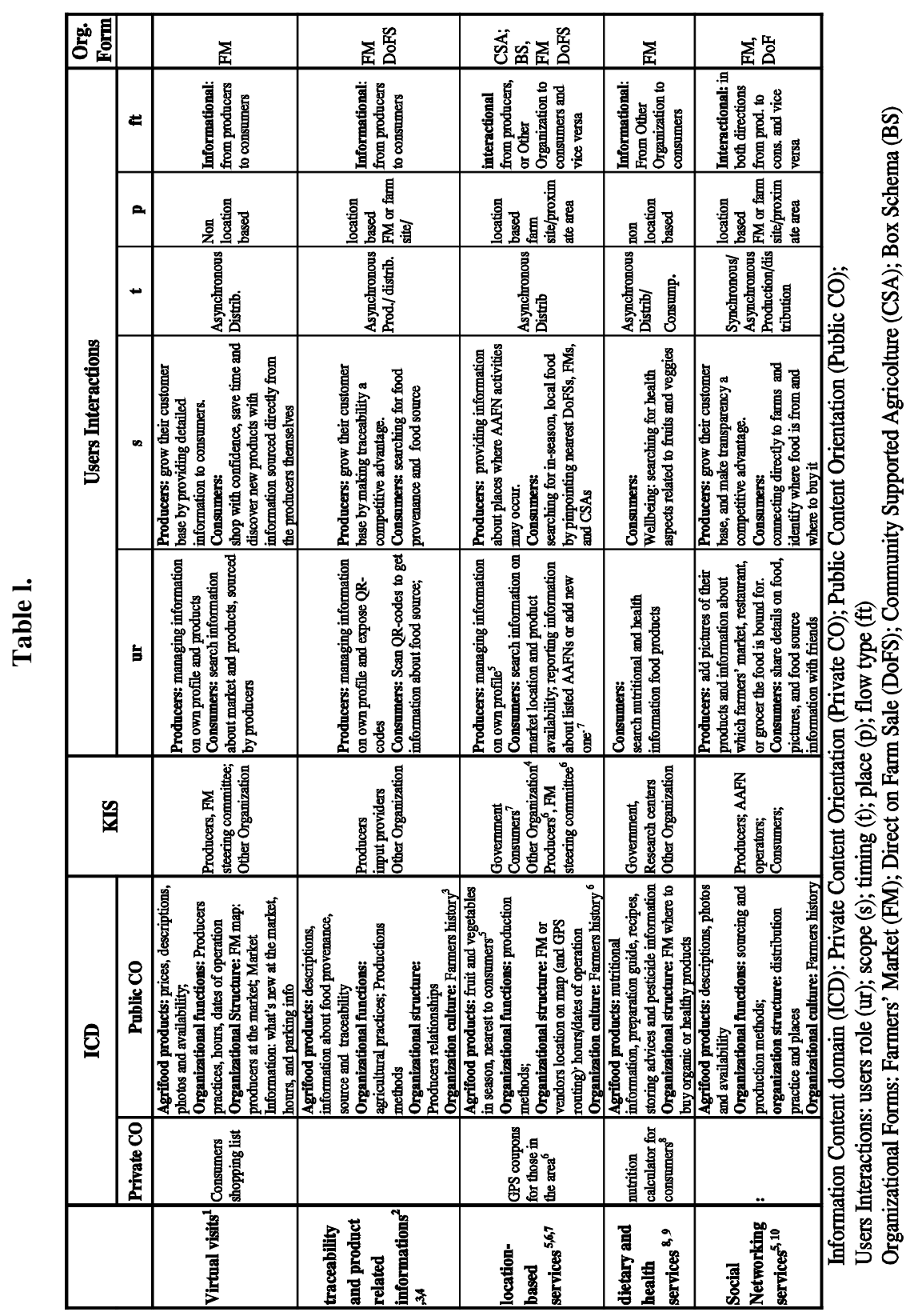




\section{Table 1. Reference urls of mobile services}

1. Edibly Pike Place Market (itunes.apple.com/us/app/edibly/id553296441?mt=8)

2. shopSavvy (shopsavvy.mobi/2011/03/08/food-traceability-on-shopsavvy; top10produce.com)

3. localsqr (localsqr.com)

4. HarvestMark Food Traceability (harvestmark.com)

5. locavore (getlocavore.com)

6. Atlantic Highlands Farmer's Ma (itunes.apple.com/us/app/atlantic-highlandsfarmers/id521136603? $\mathrm{mt}=8$ )

7. NRDC Eat Local (simplesteps.org/eat-local)

8. Smart Foods-Organic Diet Buddy (saagara.com/apps/organic-diet-buddy-app)

9. Seasonal and simple (seasonalandsimple.info)

10. foodtree (foodtree.com/you);

\section{Conclusions}

AAFNs can benefit of the use of mobile information services in several different ways. Under a user perspective, the overall value of a service is based on consumption motivations, use context criticalities, user's role in the AAFN, and information sources of the service. According to this value segmentation, we have proposed an analysis framework that represents a meaningful tool for both AAFN managers and service providers. Specifically, the first ones can benefit of using the framework to evaluate AAFN people reactions to specific mobile services and to understand which types of mobile service are likely to be tried and used in an AAFN. The second ones can benefit of using the framework to segment their customers, to assess critical success factors of their services from a customer-centric viewpoint, and to develop strategies to create value for AAFNs.

Real world cases of AAFN mobile services have been taken into account as exemplifications of our analysis scheme. However, because the focus is on general characteristics of mobile services, the framework can be used to explore or create new types of services for AAFNs.

Acknowledgement. This research work was supported by AGROMATER LAB, and DIRECT-FOOD, two projects funded by the EU and the Italian Government, both aimed to support marketing of typical agrifood products in Calabria (Italy).

\section{References}

1. Watts, D., Ilbery, B., Maye, D.: Making reconnections in agro-food geography: Alternative systems of food provision. Progress in Human Geography 29(1), 22-40 (2005)

2. Sánchez Hernández, J.L.: Alternative Food Networks: concept, typology and adaptation to the spanish context. Boletín de la A.G.E. - Asociación de Geógrafos Españoles (49), 375-380 (2009)

3. Chiffoleau, Y.: From Politics to Co-operation: The Dynamics of Embeddedness in Alternative Food Supply Chains. Sociologia Ruralis 49(3), 218-235 
4. Slee, B., Kirwan, J.: Exploring hybridity in food supply chains. In: 105th EAAE Seminar International Marketing and International Trade of Quality Food Products, Bologna, March 8-10 (2007)

5. Goodman, D.: Editorial the quality "turn" and alternative food practices: reflections and agenda. Journal of Rural Studies, 1-7 (2003)

6. Renting, H., Marsden, T., Banks, J.: Understanding alternative food network: Exploring the role of short food supply chains in rural development. Enviroment and Planning A 35, 393-411 (2003)

7. Volpentesta, A.P., Ammirato, S.: A Collaborative Network Model for Agrifood Transactions on Regional Base. In: Lytras, M.D., Ordonez de Pablos, P., Ziderman, A., Roulstone, A., Maurer, H., Imber, J.B. (eds.) WSKS 2010. CCIS, vol. 112, pp. 319-325. Springer, Heidelberg (2010)

8. Volpentesta, A.P., Ammirato, S.: Networking farmers clusters and consumers groups in local agribusiness. In: Pervasive Collaborative Networks, Boston. IFIP, pp. 33-40 (2008)

9. Ammirato, S., Della Gala, M., Volpentesta, A.P.: Alternative Agri-Food Networks as learning communities: some issues for a classification model. In: Lytras, M.D., Ruan, D., Tennyson, R.D., Ordonez De Pablos, P., García Peñalvo, F.J., Rusu, L., et al. (eds.) WSKS 2011. CCIS, vol. 278, pp. 293-300. Springer, Heidelberg (2013)

10. Volpentesta, A.P., Ammirato, S.: Alternative Agrifood Networks in a regional area: A case study. International Journal of Computer Integrated Manufacturing 26(1-2), 55-66 (2013), doi:10.1080/0951192X.2012.681911

11. Jarosz, L.: The city in the country: Growing alternative foodnetworks in Metropolitan areas. Journal of Rural Studies 24(3), 231-244 (2008)

12. Volpentesta, A.P., Ammirato, S., Della Gala, M.: Knowledge exchange and social learning opportunities in direct agri-food chains. In: Camarinha-Matos, L.M., Xu, L., Afsarmanesh, H. (eds.) Collaborative Networks in the Internet of Services. IFIP AICT, vol. 380, pp. 340-348. Springer, Heidelberg (2012)

13. Holloway, L., Kneafsey, M., Venn, L., Cox, R., Dowler, E., Tuomainen, H.: Possible Food Economies: A Methodological Framework for Exploring Food Production-Consumption Relationships. Sociologia Ruralis 47(1), 1-19 (2007)

14. Fonte, M.: Knowledge, Food and Place. A Way of Producing, a Way of Knowing. Sociologia Ruralis 48(3), 200-222

15. Heinonen, K., Pura, M.: Classifying mobile services. Sprouts: Working Papers on Information Systems 6(42) (2006), http://sprouts.aisnet.org/6-42/ (accessed March 20, 2013)

16. Clarke, I., Flaherty, T.: Mobile Portals: The Development of M-Commerce Gateways. In: Book Mobile Commerce: Technology, Theory, and Applications, pp. 185-201. Idea Group Publishing, Hershey (2003)

17. Volpentesta, A.P., Della Gala, M.: Investigating augmented learning opportunities in Direct Agri-Food. Enterprise Information Systems (submitted)

18. Sebők, A., Hegyi, A., Viola, K., Gábor, I., Homolka, F.: Food Awareness and Transparency: Current Practices and Future Tools. In: Proceedings in Food System Dynamics, pp. 639-652 (2012)

19. Parikh, T.S., Patel, N., Schwartzman, Y.: A survey of information systems reaching small producers in global agricultural value chains. In: 2nd IEEE/ACM International Conference on ICTs and Development, Bangalore, India, pp. 334-344 (2007)

20. Volpentesta, A.P., Ammirato, S., Sofo, F.: Collaborative design learning and thinking style awareness. The International Journal of Engineering Education 28(4), 948-958 (2012) 